SLAC-PUB-8120

April 1999

\title{
LASER PULSE HEATING
}

\author{
Xintian E. Lin \\ Stanford Linear Accelerator Center, Stanford University, Stanford, CA 94309
}

\begin{abstract}
Recently, interest has developed in pulsed heating effects on a copper surface [1]. Pulsed heating is one of the limits on the gradient of a structure based linac. The heat generated by an intense RF pulse on the metal surface can result in hundreds of degrees of temperature rise at $1 \mathrm{GeV} / \mathrm{m}$. After a certain number of cycles, the metal may crack due to thermal fatigue and the surface properties may deteriorate. In this article, we describe an experiment to use a high power laser to study the pulsed temperature rise on a metal surface.
\end{abstract}

Presented at the Particle Accelerator Conference (PAC99)

New York, NY, USA

March 19 - April 2, 1999

Stanford Linear Accelerator Center, Stanford University, Stanford, CA 94309

Work supported by Department of Energy contract DE-AC03-76SF00515. 


\section{Laser Pulse Heating*}

\section{Xintian E. Lin \\ Stanford Linear Accelerator Center, Stanford University, Stanford, CA 94309}

\section{Abstract}

Recently, interest has developed in pulsed heating effects on a copper surface[1]. Pulsed heating is one of the limits on the gradient of a structure based linac. The heat generated by an intense RF pulse on the metal surface can result in hundreds of degrees of temperature rise at $1 \mathrm{GeV} / \mathrm{m}$. After a certain number of cycles, the metal may crack due to thermal fatigue and the surface properties may deteriorate. In this article, we describe an experiment to use a high power laser to study the pulsed temperature rise on a metal surface.

\section{INTRODUCTION}

Laser induced damage in optical materials is a discipline in its own right. Many publications have tried to address the issue from theoretical and experimental point of view for more than 30 years[2]. However most of them concerns single or a few shots damage threshold. Thomas etc[3] has measured up to 100 shots. The damage criteria is usually a quantitative visual inspection under microscope. For a few ns and longer pulse, the threshold corresponds roughly to single shot melting of the surface. For accelerator applications, we are interested in the thermal fatigue threshold on the order of a billion shots with temperature measurement on the surface. To bypass the requirement of high power, high repetition rate microwave source, we describe an alternative experiment using a laser to test pulsed temperature rise on a copper surface.

In the geometric setting illustrated in Fig. 1, the temper-

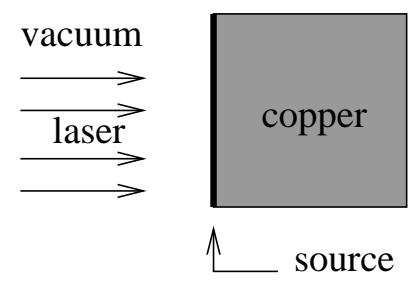

Figure 1: Surface heating.

ature rise resulting from a surface heat source induced by a laser, for example, is given by

$$
T\left(t_{p}\right)-T_{0}=\int_{0}^{t_{p}} \frac{F_{d}\left(t^{\prime}\right) d t^{\prime}}{\sqrt{\pi K \rho C_{v}\left(t_{p}-t^{\prime}\right)}},
$$

where $K, \rho$ and $C_{v}$ are the thermal conductivity, density and heat capacity respectively. The initial temperature is

\footnotetext{
${ }^{*}$ Work supported by U.S. Department of Energy, contract DE-AC03$76 \mathrm{SF} 00515$
}

denoted by $T_{0}$. If we assume that $F_{d}(t)$, the power flux deposited into the material, is a square pulse in time, then

$$
T\left(t_{p}\right)-T_{0}=F_{d} \frac{2 \sqrt{t_{p}}}{\sqrt{\pi K \rho C_{v}}} .
$$

The temperature rises as one half power of the pulse length, a generic character of heat diffusion.

In the case of a Gaussian pulse profile, the maximum temperature rise on the surface becomes

$$
T_{\max }-T_{0}=0.783 \frac{F}{t_{p}} \frac{2 \sqrt{t_{p}}}{\sqrt{\pi K \rho C_{v}}}
$$

where $F$ is the fluence deposited into the copper, and $t_{p}$ is the FWHM of the pulse. Compared to a square pulse with fluence $F$ and pulse length $t_{p}$, the lower temperature rise of Gaussian pulse is a result of the lower peak power and the spread of energy.

When the temperature rise $T$ is high, the dependence of material property on temperature needs to be considered, thus Eq. 1 becomes[1]

$$
\begin{aligned}
T\left(t_{p}\right)-T_{0} & =\int_{-\infty}^{t_{p}} \frac{F_{d}\left(t^{\prime}, T\left(t^{\prime}\right)\right) d t^{\prime}}{\sqrt{\pi K\left(T\left(t^{\prime}\right)\right) \rho C_{v}\left(T\left(t^{\prime}\right)\right)\left(t_{p}-t^{\prime}\right)}} \\
& =\int_{-\infty}^{t_{p}} \frac{f\left(t^{\prime}, T\left(t^{\prime}\right)\right) d t^{\prime}}{\sqrt{t_{p}-t^{\prime}}} .
\end{aligned}
$$

Because of the singularity at $t^{\prime}=t_{p}$, the integral may be evaluated as[4]

$$
T\left(t_{n}\right)-T_{0}=\sum_{j=-\infty}^{n-1} 2\left(\sqrt{t_{n}}-\sqrt{t_{j}}\right) f\left(t_{j}, T\left(t_{j}\right)\right) .
$$

If one were to scale time $t$ by a factor $\beta$, i.e. $\hat{t} \rightarrow \beta t$ and flux by $\sqrt{\beta}$, i.e. $\hat{F}_{d} \rightarrow F_{d} / \sqrt{\beta}$, then from Eq. 4 ,

$$
\hat{T}(\beta t)-T_{0}=T(t)-T_{0}
$$

regardless of the temperature dependence of the material property. Thus to get the same temperature rise, $F / \sqrt{t_{p}}$ needs to be constant.

\section{LASER ABSORPTANCE IN METAL}

Shining a laser with wavelength $\lambda$ perpendicularly onto a copper surface, the reflection coefficient can be expressed as

$$
r=\frac{n-1}{n+1}
$$

where the refractive index $n=\sqrt{\epsilon}$. The Drude model is quite adequate in infrared[5]. It gives

$$
\epsilon=1-\frac{\omega_{p}^{2}}{\omega^{2}+i \omega \omega_{\tau}} .
$$


The plasma frequency $\omega_{p}^{2}=n_{e} e^{2} / m \epsilon_{0}$, and the collision frequency $\omega_{\tau}=n_{e} e^{2} / m \sigma(T)$. The electron density $n_{e}$ inside copper is roughly $8.4 \times 10^{22} / \mathrm{cm}^{3}$. Notice that electric conductivity $\sigma$ is temperature dependent. From energy conservation, the flux into copper has the form

$$
F_{d}=\left(1-|r|^{2}\right) I_{0},
$$

where $I_{0}$ is the laser irradiance. In the frequency region $\omega_{\tau} \ll \omega \ll \omega_{p}$, i.e. $0.11 \mu m \ll \lambda \ll 46 \mu m$, the Drude model gives

$$
F_{d} \approx \frac{2 \omega_{\tau}}{\omega_{p}} I_{0}
$$

The absorptance is wavelength independent. So the following experiment may be carried out at other wavelengths with about the same temperature rise.

When laser wavelength approaches $1 \mu \mathrm{m}$, other contribution becomes important[8], and analysis becomes more involved.

\section{EXPERIMENT}

The experiment, illustrated in Fig. 2, utilizes high energy laser pulses to heat up the sample surface repeatedly and uses interference between the reflected pulse and original pulse to monitor the surface temperature. Mirror 1 and 2

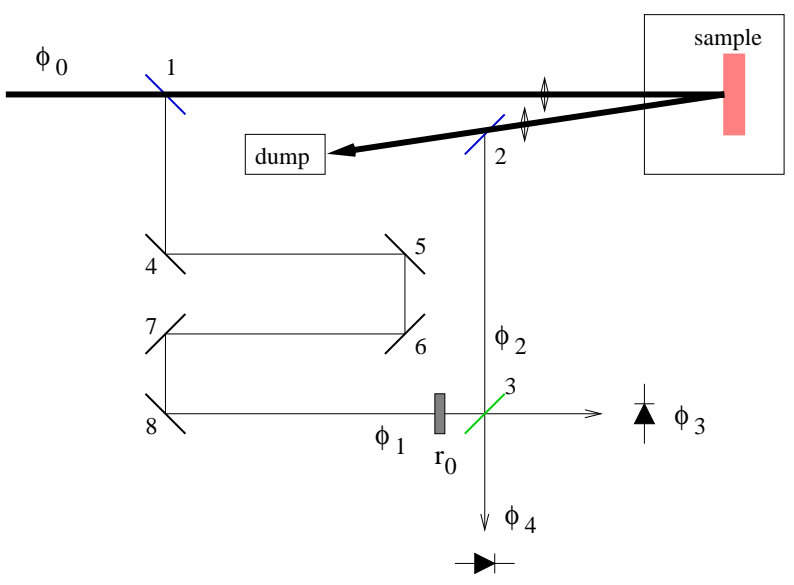

Figure 2: Laser pulse heating experiment.

are beam splitters and mirror 3 is a combiner such that the wave functions $\phi$ 's are given by

$$
\left.\begin{array}{rl}
\phi_{1} & =\alpha \phi_{0} \\
\phi_{2} & =r \alpha \phi_{0} \\
\phi_{3} & =\frac{1}{\sqrt{2}}\left(r_{0} \phi_{1}-\phi_{2}\right)=\alpha \phi_{0} \frac{r_{0}-r}{\sqrt{2}} \\
\phi_{4} & =\frac{1}{\sqrt{2}}\left(r_{0} \phi_{1}+\phi_{2}\right)=\alpha \phi_{0} \frac{r_{0}+r}{\sqrt{2}}
\end{array}\right\}
$$

where we have assumed the splitting factor $\alpha \ll 1$. A phase shifter/attenuator $r_{0}$ is inserted in $\phi_{1}$ to insure null reading of $\phi_{3}$ at low power.

At $10.6 \mu \mathrm{m}$ wavelength, $|r|$ is very close to 1 . Therefore, $\phi_{4}$ is a good measure of the pulse energy and $\phi_{3}$ is a measure of energy loss to the surface, and thus gives the surface temperature through the $\sigma(T)$ dependence.
The temperature dependence of the copper properties $K$ and $\sigma$ are tabulated in [6], and a polynomial fit is used to interpolate to other temperatures. The heat capacity $C_{v}$ of copper is given in [7].

Taking $\lambda=10.6 \mu \mathrm{m}$, a square pulse with $t_{p}=16 \mathrm{~ns}$ and $I_{0}=1.5 \mathrm{GW} / \mathrm{cm}^{2}$ for example, the pulsed temperature rise is as high as $991^{\circ} \mathrm{C}$. The surface temperature, plotted in Fig. 3, has an initial value of $300 \mathrm{~K}$. The solid

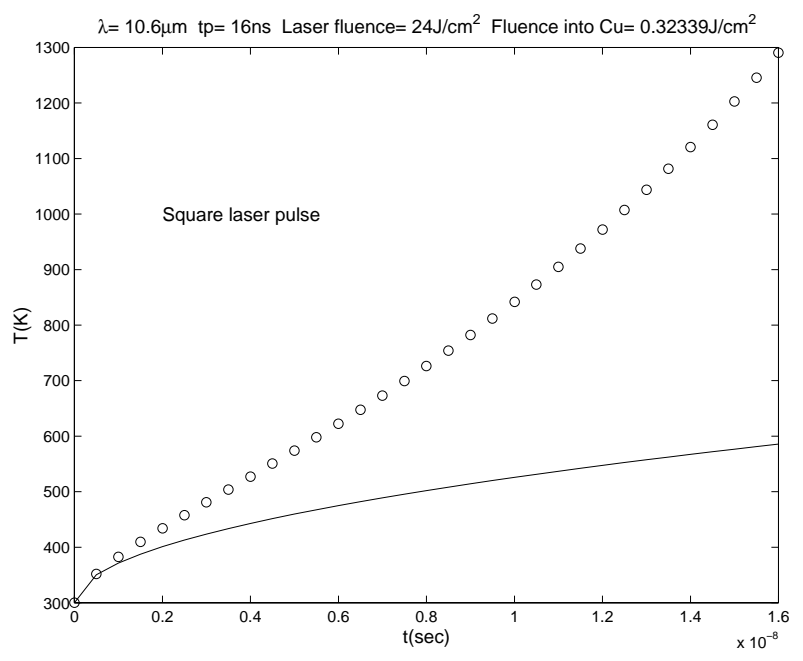

Figure 3: Pulsed temperature rise. The solid curve is estimation from Eq. 2 and circles are from Eq. 4.

curve is calculated from Eq. 2. The circles, with temperature dependence of the material property included (Eq. 4), depart significantly from Eq. 2 at higher temperatures. The faster temperature growth is primarily due to higher electric resistivity at higher temperature. The heat capacity and thermal conductivity, on the other hand, change less than $20 \%$ in this temperature range.

In the case of a comparable Gaussian pulse, the maximum temperature rise, illustrated in Fig. 4 , is about $556^{\circ} \mathrm{C}$, $44 \%$ lower than the square pulse case. The rest of the discussions all concern Gaussian pulses.

The temperature rise $T_{\text {max }}$ and diode outputs $\int\left|\phi_{3}\right|^{2} d t$, $\int\left|\phi_{4}\right|^{2} d t$ are plotted in Fig. 5 and 6 as a function of laser fluence. The maximum temperature rise $T_{\max }$, plotted against the signal $s=\int\left|\phi_{3}\right|^{2} d t / \int\left|\phi_{4}\right|^{2} d t$, is illustrated in Fig. 7. A least square fit given by

$$
s=6.83 \times 10^{-7}\left(1-2.31 \frac{T_{\max }}{300}+1.273\left(\frac{T_{\max }}{300}\right)^{2}\right)
$$

is also plotted. It is the basis of surface temperature monitoring.

\section{CONCLUSION}

This experiment can be carried out using $10.6 \mu \mathrm{m} \mathrm{CO}_{2}$ laser with $2.4 \mathrm{~mJ}$ in $16 \mathrm{~ns}$ pulse running at $1 \mathrm{kHz}$. The laser needs to be focused to a $100 \mu \mathrm{m}$ spot to deliver $24 \mathrm{~J} / \mathrm{cm}^{2}$ fluence. It is then able to test surface temperature rise up to $556^{\circ} \mathrm{C}$ reaching $10^{9}$ pulses in 2 weeks. To see a 100 degree 


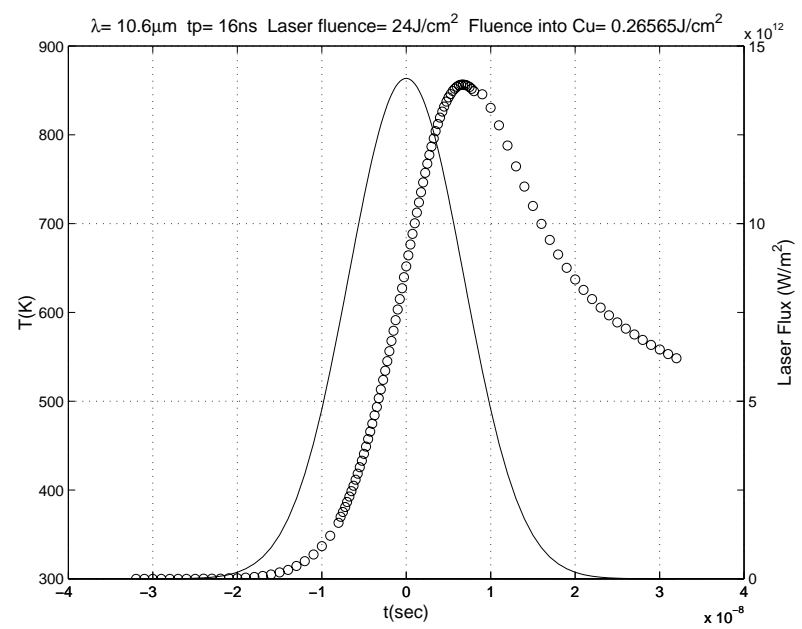

Figure 4: Pulsed temperature rise for a Gaussian laser pulse. The solid line represents the laser irradiance, and the surface temperature is drawn in circles.

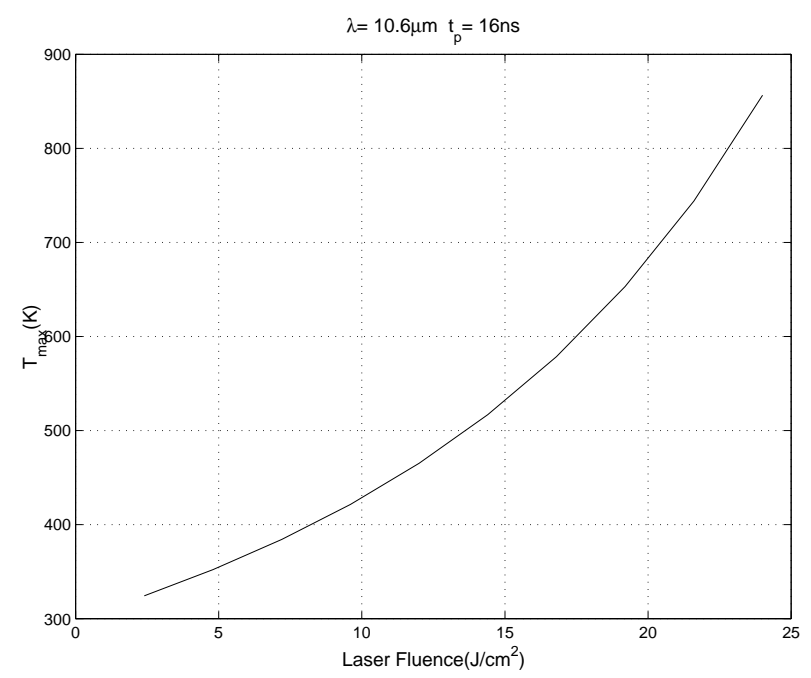

Figure 5: Maximum pulsed temperature rise.

temperature rise, a $2 \mathrm{~nJ}$ diode detector running at $1 \mathrm{~Hz}$ rate suffices. The drift in diode reading is an indicator of the surface degradation. The same experiment, carried out at other wavelengths, $1 \mu \mathrm{m}$ or $100 \mu \mathrm{m}$ for example, are also desirable to check the frequency dependence. The laser fluence requirement is about the same. The spot size may need to be increased to alleviate focusing requirement at the longer wavelength. The data analysis is more involved at shorter wavelength because the interband contribution to absorptance is significant[8].

\section{REFERENCES}

[1] D. Pritzkau, et al. "Experimental study of pulsed heating of electromagnetic cavities", 1997 IEEE PAC, Vancouver, Candana, 12-16 May 1997.

[2] Laser Induced Damage in Optical Materials, NIST and SPIE publications. 1969-
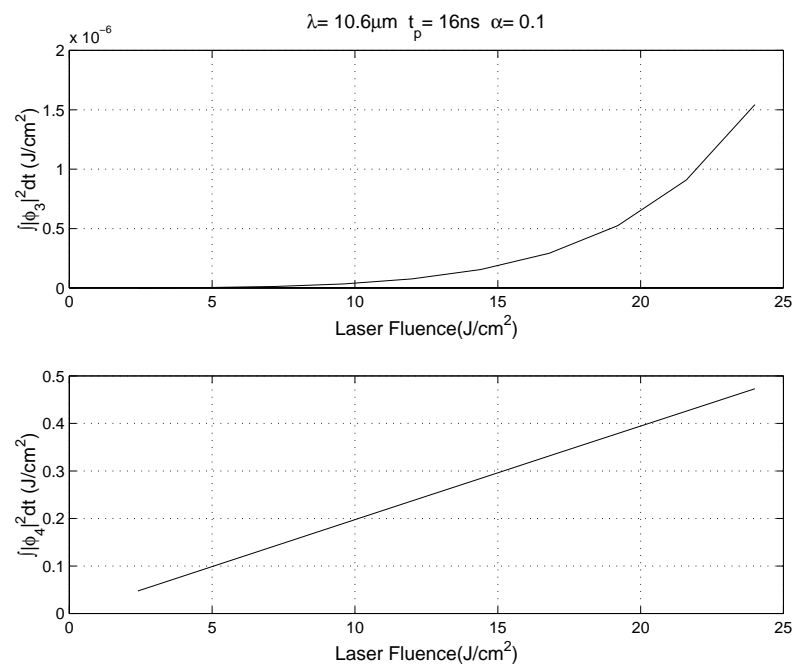

Figure 6: The integrated diode signals as a function of laser fluence.

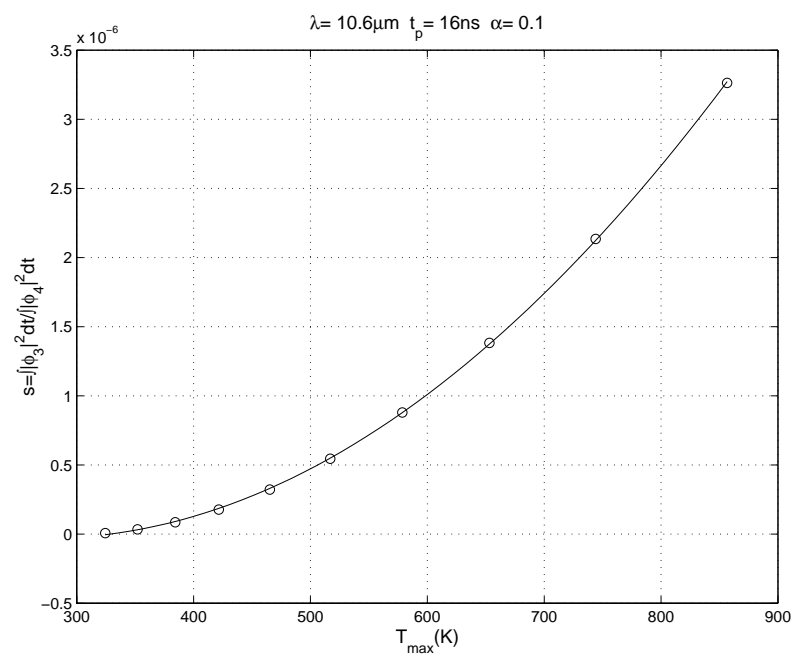

Figure 7: The signal $s=\int\left|\phi_{3}\right|^{2} d t / \int\left|\phi_{4}\right|^{2} d t$ plotted as a function of the maximum surface temperature rise $T_{\max }$. The circles are the result of Eq. 4 and 11. The solid line is a quadratic least square fit.

[3] S.J. Thomas, R.F. Harrison and J.F. Figueira. "Observations of the morphology of laser-induced damage in copper mirrors", Appl. Phys. Lett. 40, 200(1982).

[4] P. Linz, "Analytical and Numerical Methods for Volterra Equations", 1985, Eq.(8.15)

[5] CRC handbook of Laser Science and Technology Volume IV, Boca Raton, Fla. 1986.

[6] David R. Lide, "Handbook of Chemistry and Physics" 77th edition. 1997 p12-40, p12-174.

[7] G. T. Furukawa and T. B. Douglas, "American Institute of Physics Handbook", 3rd edition. p4-113

[8] M. Sparks and E. Loh, Jr., "Temperature dependence of absorptance in laser damage of metallic mirrors: I. Melting", J. Opt. Soc.Am., Vol 69, 1979, 847-858. 